\title{
MHD Casson non-Newtonian nanofluid over a nonlinear penetrable elongated sheet with thermal radiation and chemical reaction
}

\author{
Seethamahalakshmi VYAKARANAM ${ }^{1}$, Venkata Ramana Reddy GURRAMPATI ${ }^{2, *}$ and \\ Y Hari Krishna ${ }^{3}$ \\ ${ }^{1}$ Department of Mathematics, PVP Siddartha Institue of Technology, Kanur,India-520007. \\ ${ }^{2}$ Department of Mathematics, Koneru Lakshmaiah Education Foundation, Vaddeswaram-522502. \\ ${ }^{3}$ Department of Mathematics, ANURAG Engineering College, Anathgiri (v), Kodad, Suryapet, Telangana, India-508206.
}

\begin{abstract}
Consider a steady flow in two-dimensional of a viscous, incompressible Casson nano liquid over a nonlinear penetrable elongated sheet with radiation and chemical reaction. The Casson liquid rheological model is used to explain the non-Newtonian liquid attributes. Similarity variables are utilized to evaluate the governing flow model into set of nonlinear total differential equations. The outcomes of the flow equations were gotten by using Runge-Kutta alongside the shooting techniques. In other to explain the physics of the problem, impact of flow parameters are presented in graphs while computations on engineering curiosity are presented in table. Ahike in the Casson liquid term is observed to degenerate the fluid velocity alongside the momentum layer thickness. The impact of the imposed magnetic is felt by decreasing the velocity owning to the Lorentz force.
\end{abstract}

Keywords: Non-Newtonian fluid, elongated sheet, Lorentz force, thermal radiation, Chemical reaction.

\section{Introduction}

Fluids are generally classified as Newtonian and non-Newtonian. The Newtonian fluids signifies the linear relationship that exist between shear stress as well as shear rate. On the contrary, nonNewtonian fluids portrays the nonlinear relationship existing between the shear stress and strain. Examples of Newtonian fluids are water, oil etc while Casson, Walters-B liquid, micropolar, power law liquids etc are examples on non-Newtonian liquids. One of the most popular non-Newtonian liquid is Casson liquid. It finds applications in the manufacturing of food, biological engineering and in drilling operations. Mahanta and Shaw [1] elucidate the flow of Casson liquid in 3D past a penetrable linearly stretchable sheet. Samrat et al. [2] elucidate the attribute of thermal radiation with unsteadiness flow of Casson nano liquid past a stretchable surface. Raza et al. [3] studied the impact of multiple slip on MHD non-Newtonian nano liquid flow past an elongated sheet. Mukhopadhyay et al.[4] gave detailed behaviour of Casson liquid over a stretchable surface. The flow of Casson liquid with MHD past an exponentially and shrinking sheet has been elucidated by Nadeem et al. [5]. Vijaya and Reddy [6] discussed Casson liquid flow past an upward penetrable plate with radiation as well as Soret impact. Krishna et al. [7] elucidate chemically reactive MHD flow of Casson fluid with penetrable stretchable sheet. The recent study of Idowu and Falodun [8] discussed the simultaneous flow of two nonNewtonian liquids by varying viscosity as well as thermal conductivity. In another study of Idowu and Falodun [9] presented discussion on MHD non-Newtonian nano liquid past a slanting plate with thermophoresis. Idowu et al.[10] studied Casson dissipative liquid by varying viscosity alongside thermal conductivity.

The thermal radiation acts a major role in industrial engineering. It major importance on heat transport process is in design of energy system utilized at a very higher temperature. Examples are missiles, gas turbines, space vehicles, nuclear power plants and satellites. Fagbade et al. [11] employed spectral approach to the problem of MHD flow of Walters-B liquid with thermal radiation alongside heat source. 
Vedavathi et al. [12] elucidate radiation with mass transport impact on unsteadiness flow of MHD over an infinite upward plate. Manglesh and Gorla [13] explored unsteadiness of MHD viscoelastic slip motion with thermal radiation alongside chemically reactive rotating channel. Ullah et al. [14] extensively discussed the unsteadiness of MHD Casson liquid with thermal radiation and situated in a penetrable channel. Bala [15] elucidate Casson liquid attribute with MHD flowing through an exponentially slanting penetrable surface with thermal radiation as well as chemical reaction. Chemical reaction effects on heat alongside mass transport by mixed convection laminar flow in an upward surface with induced magnetic field and radiation is discussed by Ibrahim et.al [16]. Vedavathi et.al [17] has analyzed the heat transport on MHD nano liquid flow past a half-infinite flat plate folded in a penetrable channel with radiation absorption, heat source and diffusion thermo effect. Sridhar et.al [18] investigated the numerical techniques of the flow and heat transport of Casson fluid over an exponentially pores stretchable surface with thermal radiation.Numerical study of diffusion flow on a species reacting chemically over the flow on MHD exponentially stretchable surface of a Casson liquid by Kumari et.al.[19]. Sree Devi et.al [20] studied the effect of radiation and radiation absorption on convective heat and mass transport flow of a viscous as well as electrically conducting liquid in a non-uniformly heated vertical channel. Arundhati et.al [21] used the non-Darcy convective heat plus mass transport flow with pores in vertical channel with Soret, Dufour and chemical reaction effects. Later, Chandra Sekhar et.al [22] examined the heat transfer analysis of second grade liquid past a stretchable sheet through channels with pores under chemical reaction influence. Konijeti et.al [23] investigated of the MHD mixed convection flow of radiating and reacting chemically Casson nanoliquid past a nonlinear stretchable sheet with viscous dissipation and heat source. Vijaya et.al
[24] elucidates the boundary layer flow of a mixed convective nanoliquid past an upward circular cylinder under magnetic field, heat radiation and external surface temperature imfluence. Suneetha et al. [25] studied free convective heat along with mass transport flow in a penetrable channel with thermal radiation and reacting chemically. Alao et al.[26] elucidate the behaviour of thermal radiation of heat and mass transport and chemically reactive liquid numerically.

The consideration of heat alongside mass transport with MHD have received much attention by many scholars owning to their numerous applications in chemical engineering and sciences. Liquids such as ethylene glycol, water and oil are poor conductivity of heat, they are found very useful as a cooling materials for boosting manufacturing. The presence of MHD drags the flow owning to the production of Lorentz force. This indicate the usefulness of MHD in reducing the turbulence of fluid flow.

Inspired by the literature discussed above to study a steady two-dimensional flow of a viscous, incompressible non-Newtonian nano liquid over a nonlinear penetrable elongated sheet with radiation and chemical reaction. Similarity transformation has been utilized to simplify the governing equations, and the reduced boundary value problems are solved using Runge-kutta method alongside shooting techniques.

\section{Mathematical analysis}

Consider steady, laminar, viscous and incompressible boundary layer flow of nano liquid past a nonlinear penetrable and stretchable surface. The $y$-coordinate is taken normal to the stretching surface while the flow occurs immediately $y \leq 0$. The Casson liquid flow arises owning to the stretching sheet that take place out of the shit at $x=0$ and $y=0$. Let us assume that a certain point at the plate, the speed is equivalent to the distance from the slit. Thus, the approximation of the boundary layer is valid on the flow. Starting from the wall where $U_{w}=a x^{n}$, the sheet is assumed 
to vary non-linearly with a distance of $x$. In the wall velocity $U_{w}=a x^{n}$, ' $a$ ' signifies constant, that is $a>0$ while $n$ signifies nonlinear stretching term. According to the constitutive equation of Casson liquid as described as follows:

$$
\tau_{i j}=\left\{\begin{array}{l}
\left(\mu_{b}+\frac{P_{y}}{\sqrt{2 \pi}}\right) 2 e_{i j} \text { when } \pi>\pi_{c} \\
\left(\mu_{b}+\frac{P_{y}}{\sqrt{2 \pi_{c}}}\right) 2 e_{i j} \text { when } \pi<\pi_{c}
\end{array}\right.
$$

where $P_{y}$ signifies Casson yield stress expressed as

$$
P_{y}=\frac{\mu_{b} \sqrt{(2 \pi)}}{\beta}
$$

$\mu_{b}=$ plastic dynamic viscosity, $\pi=e_{i j} e_{i j}=$ multiplication of the component of deforming rate with itself and $e_{i j}=$ deformation rate and $\pi_{c}=$ critical value subject to Casson model. The Casson liquid flow where $\pi>\pi_{c}$, we have

$$
\mu_{0}=\mu_{b}+\frac{P_{y}}{\sqrt{2 \pi}}
$$

putting equation (2) into (3), thus kinematic viscosity is subject to plastic dynamic viscosity $\mu_{b}$ , the density $\rho$ and Casson term $\beta$ gives

$$
\mu_{0}=\frac{\mu_{b}}{\rho}\left(1+\frac{1}{\beta}\right)
$$

Putting into consideration all the assumptions, the flows equations that govern the model are as follows:

$$
\frac{\partial u}{\partial x}+\frac{\partial v}{\partial y}=0
$$

$u \frac{\partial u}{\partial x}+v \frac{\partial u}{\partial y}=\frac{\mu}{\rho}\left(1+\frac{1}{\beta}\right) \frac{\partial^{2} u}{\partial y^{2}}-\frac{\sigma B_{0}^{2}}{\rho} u-\frac{\mu_{b}}{k \rho}\left(1+\frac{1}{\beta}\right) u$

$$
u \frac{\partial T}{\partial x}+v \frac{\partial T}{\partial y}=\alpha \frac{\partial^{2} T}{\partial y^{2}}+\tau\left[D_{B} \frac{\partial C}{\partial y} \frac{\partial T}{\partial y}+\frac{D_{T}}{T_{\infty}}\left(\frac{\partial T}{\partial y}\right)^{2}\right]-\frac{1}{\rho c_{p}} \frac{\partial q_{r}}{\partial y}+\frac{\mu}{\rho c_{p}}(1
$$

$$
u \frac{\partial C}{\partial x}+v \frac{\partial C}{\partial y}=D_{B} \frac{\partial^{2} C}{\partial y^{2}}-k_{l}\left(C-C_{\infty}\right)+\frac{D_{T}}{T_{\infty}} \frac{\partial^{2} T}{\partial y^{2}}
$$

subject to:

$$
u=U_{w}+S_{1} \frac{\partial u}{\partial y}, v= \pm v_{w}, T=T_{w}+S_{2} \frac{\partial T}{\partial y}, C=C_{w}+S_{3} \frac{\partial C}{\partial y}
$$

$$
u \rightarrow 0, ; T \rightarrow T_{\infty}, C \rightarrow C_{\infty}, \text { as, } y \rightarrow \infty
$$

here $u$ and $v$ are velocity components in $\mathrm{x}$ and $\mathrm{y}$ direction, $\quad v$ signifies viscosity, $\beta$ signifies Casson term, $\sigma$ signifies electrical conductivity, $B_{0} \quad$ signifies magnetic constant, $\rho$ signifies density, $\mathrm{K}$ signifies porosity term, $\mathrm{T}$ signifies temperature, $\alpha$ signifies thermal conductivity, $\tau$ signifies heat capacity ratio, $D_{B}$ signifies mass diffusivity, $\mathrm{C}$ signifies concentration, $D_{T}$ signifies thermophoretic diffusion coefficient, $T_{\infty}$ signifies ambient temperature, $\mu$ signifies coefficient of viscosity, $c_{p}$ signifies specific heat, $q_{r}$ signifies radiative heat flux, $k_{l}$ signifies chemical reaction term, $S_{1}, S_{2}$ and $S_{3}$ signifies slip terms respectively.

To simplify the radiative heat flux on the flow, application of Rosseland diffusion approximation is preference as reported in Alao et al.[25] and Fagbade et al.[27] such that:

$$
q_{r}=-\frac{4 \sigma_{s}}{3 k_{e}} \frac{\partial T^{4}}{\partial y}
$$

From the above, the Stefan-Boltzman constant is $\sigma_{s}$ and the mean absorption coefficient is $k_{e}$. Let surmised that the difference in temperature in the flow are sufficiently small so that $T^{4}$ may be expressed in a linear form by expanding $T^{4}$ about 
$T_{\infty}$ using Taylor series and neglecting higher terms to obtain

$$
T^{4} \neg 4 T_{\infty}^{3} T-3 T_{\infty}^{4}
$$

substituting (12) into (11) and the result to the third term of the energy eqn to obtain

$$
-\frac{\partial q_{r}}{\partial y}=\frac{16 \sigma_{s} T_{\infty}^{3}}{3 k_{e}} \frac{\partial^{2} T}{\partial y^{2}}
$$

The similarity transformation defined as follows are employed in this study

$u=a x^{n} f^{\prime}(\eta), v=-a x^{\frac{n-1}{2}} \sqrt{\frac{v}{a}}\left(\frac{n+1}{2} f(\eta)+\frac{n-1}{2} \eta f^{\prime}(\eta)\right)$,

$\eta=\sqrt{\frac{a}{v}} x^{\left(\frac{n-1}{2}\right)} y, \theta(\eta)=\frac{T-T_{\infty}}{T_{w}-T_{\infty}}, \phi(\eta)=\frac{C-C_{\infty}}{C_{w}-C_{\infty}}$

With respect to equation (14) above, the governing flow equations are simplified to obtain

$\left(1+\frac{1}{\beta}\right) f^{\prime \prime \prime}+\frac{n+1}{2} f f^{\prime \prime}-n\left(f^{\prime}\right)^{2}-\left(M+\left(1+\frac{1}{\beta}\right) \frac{1}{K}\right) f^{\prime}=0$

$\left(\frac{1+R}{P r}\right) \theta^{\prime \prime}+\frac{n+1}{2} f \theta^{\prime}-n f^{\prime} \theta+N b \theta^{\prime} \phi^{\prime}+N t\left(\theta^{\prime}\right)^{2}+\operatorname{PrEc}\left(1+\frac{1}{\beta}\right) f^{\prime \prime}=0$

$$
\phi^{\prime \prime}+\left(\frac{n+1}{2}\right) \operatorname{Lef} \phi^{\prime}+\frac{N t}{N b} \theta^{\prime \prime}-K r \phi=0
$$

with the constraints:

$f(0)=f_{w}, f^{\prime}(0)=1+L_{1} f^{\prime \prime}(0), \theta(0)=1+L_{2} \theta^{\prime}(0), \phi(0)=1+L_{3} \phi^{\prime}(0)$

$$
f^{\prime}(\infty) \rightarrow 0, \theta(\infty) \rightarrow 0, \phi(\infty) \rightarrow 0
$$

Obviously, $n$ signifies nonlinear stretching parameter while $\mathrm{x}$ signifies coordinate along the surface towards $f_{w}$ and $L_{1}$. To simplify $f_{w}$ as well as $L_{1}$ in a dimensionless form, $P_{n x}=\left(\frac{x^{n-1}(n+1)}{2}\right)$ is employed. Hence, $f_{w}$ together with $L_{1}$ is subject to $P_{n x}$ as

$$
F_{w}=\frac{f_{w}}{\sqrt{P_{n x}}}, L_{1}=S \sqrt{P_{n x}}
$$

The engineering curiosity are skin friction $\left(C_{f}\right)$, local Nusselt $(\mathrm{Nu})$ and Sherwood $(\mathrm{Sh})$ defined as follows:

$$
C_{f}=\frac{\tau_{w}}{\rho u_{w}^{2}}, N u=\frac{x q_{w}}{K\left(T_{w}-T_{\infty}\right)}, S h=\frac{x q_{m}}{D_{B}\left(C_{w}-C_{\infty}\right)}
$$

and $\tau_{w}$ signifies wall shear stress, $q_{w}$ signifies heat flux while $q_{m}$ signifies mass flux. They are

$$
\tau_{w}=\mu_{B}\left(1+\frac{1}{\beta}\right)\left(\frac{\partial u}{\partial y}\right)_{y=0}, q_{w}=-K\left(\frac{\partial T}{\partial y}\right)_{y=0}, q_{m}=-D_{B}\left(\frac{\partial C}{\partial y}\right)_{y=0}
$$

Employing equation (14) on the above to obtain

$$
R e_{x}^{\frac{1}{2}} C_{f}=\left(1+\frac{1}{\beta}\right) f^{\prime \prime}(0), R e_{x}^{\frac{1}{2}} N u=-\theta^{\prime}(0), R e_{x}^{\frac{1}{2}} S h=-\phi(0)
$$

\section{Numerical approach}

The converted nonlinear differential Equations (15)-(17) with the boundary conditions (18) and (19) are elucidated by Runge-Kutta Fehlberg method along with shooting technique. This method has been proven to be adequate and gives accurate results for the boundary layer equations

\section{Results and Discussion}

This section explains the outcomes of the results from solving equation (15)-(17) subject to (18) and (19) numerically. To describe the physics of the problem, results are presented in graphs. Figure 2 portrays the effect of porosity term (K) on the velocity plot. It is noticed that an incremental value of $\mathrm{K}$ leads to degeneration in the velocity plot as well as the momentum layer thickness. Physically, a hike in K makes very hard for the liquids particles to move freely and hereby resist the velocity gradually. Figure 3 portrays the behaviour of the imposed magnetic term (M) on the velocity plot. An increment in the value of $M$ as shown in Figure 3 declines the velocity plot. Practically, imposing the magnetism in the flow direction of an electrically conducting liquid such as Casson produces Lorentz force. This force has 
the tendency to drag the Casson flow by decreasing its velocity. hence, the fluid velocity alongside its momentum layer thickness is declined. This implies that, increase in $\mathrm{M}$ added more strength to the Lorentz force.

Figure 4 presents the impact of varying the nonlinear stretching term (n) on the velocity plot. Obviously from figure 4, an increment in the stretching term degenerates the velocity plot alongside the momentum layer thickness. Figure 5 portrays the impact of the Casson $(\beta)$ electrically conducting liquid on the velocity plot. A large value of $\beta$ is detected to degenerate the velocity plot from 1 to 4 . This indicates that the density alongside momentum layer thickness declines for a large value of $\beta$. Experimentally, when $\beta \rightarrow \infty$ it is detected that the behaviour of $\beta$ is equivalent to a Newtonian behaviour. Physically, the Casson liquid possesses a plastic dynamic viscosity originates a resistance to the liquid flow by decreasing the velocity plot.

Figure 6 portrays the role of thermal radiation $(R)$ on the temperature plot. A large value of $\mathrm{R}$ is observed to hike the temperature plot. This implies an enhancement in the thermal layer thickness. Physically, the thermal radiation helps to boost the thermal situation of the liquid environment. Hence, increasing $\mathrm{R}$ helps to boost the heat on the liquid and hereby lead to enhancement in the temperature of the liquid. Figure 7 represents the impact of the Prandtl number (Pr) on the temperature plot. A less fluid temperature alongside thermal layer thickness is noticeable for a large Pr. Hence, when $\operatorname{Pr} \ll 1$, it implies thermal diffusivity controls the flow behavior while implies momentum diffusivity controls the flow behavior. Therefore, is useful in controlling cooling rate of a conducting liquid electrically. In this study, there exist a wall temperature and free stream temperature which gives rise to $T_{w}-T_{\infty}$ along with thermal boundary layer thickness.
Figure 8 shows the effect of Eckert number $(E c)$ on the temperature plot. An increment in the value of $E c$ from 0.1 to 0.4 is observed to degenerate the temperature plot alongside the thermal layer thickness. Ec portray the flow kinetic energy and its associated enthalpy. The viscous dissipation term (Eckert number) hike heat energy to the fluid environment. Physically, Eckert number and thermal radiation in a fluid flow increase the rate of heat transfer. Now, considering Eckert number, thermal radiation with thermally-stratified medium assist to boast the heat transport rate by elevating the thickness of thermal boundary layer. Figure 9 represents the effect of the thermophoresis $(N t)$ on the concentration plot. It is detected in figure 9 that a large value of $N t$ hike the heat transport rate by enhancing the fluid temperature plot.

Figure 10 depicts the effect of the Brownian motion $(\mathrm{Nb})$ on the concentration plot. A large value of $N b$ is observed to declines the concentration plot as well as the solutal layer thickness. The result in figure 10 shows the impact of $N b$ on concentration plot is opposite to that of $N t$. Figure 11 shows the impact of the chemical reaction term $(K r)$ on the concentration plot. An incremental value of $R$ is observed to degenerate the concentration plot. Physically, it indicate a destructive chemical reaction. Figure 12 represents the effect of the Lewis number ( $L e$ ) on the concentration plot. An increment in $L e$ is observed in figure 12 to degenerate the concentration plot. Figure 13 shows the slip effect on the velocity profile. The result in figure 13 shows a decrease in the velocity plot owning to a hike in the slip term.Figure 14 shows the slip effect on the temperature profile. The result in figure 14 shows a decrease in the temperature plot due to increase in the slip term. Figure 15 shows the slip effect on the species plot. The result in figure 15 shows a decrease in the concentration plot due to increase in the slip term. Table 1 shows the impact of various flow parameters on 
the engineering interest. All pertinent flow parameters were found to have great effect on the

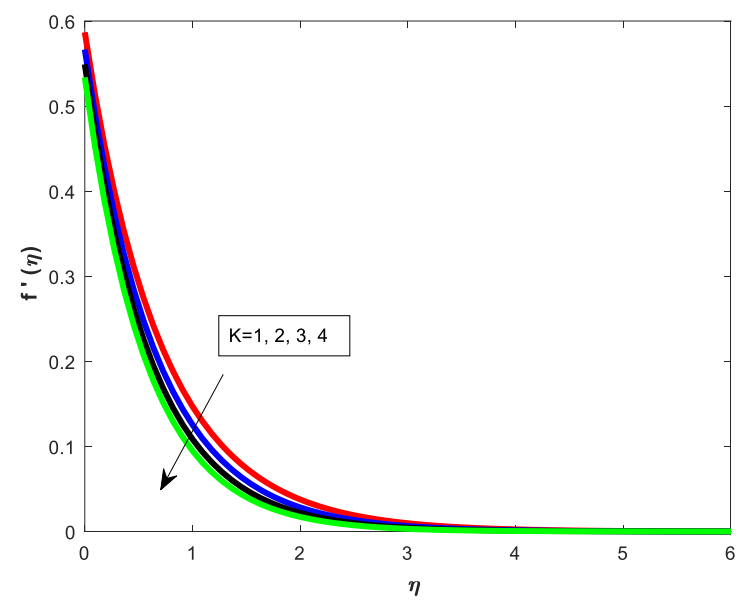

Figure 2: Effect of $\mathrm{K}$ on velocity profile

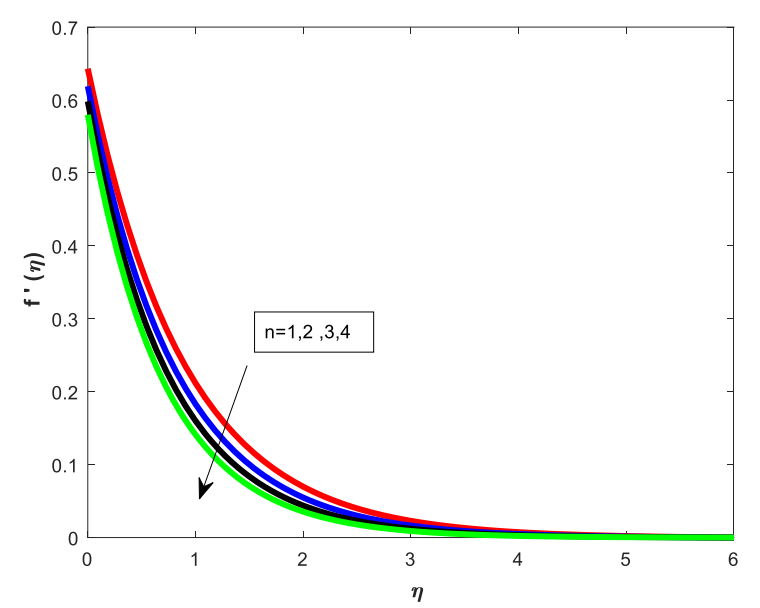

Figure 4: Effect of $\mathrm{n}$ on velocity profile

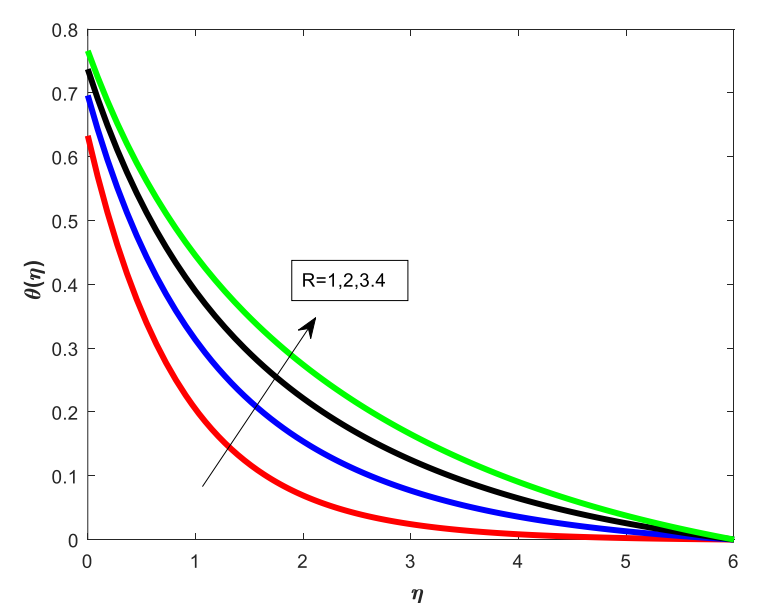

Figure 6: Effect of R on temperature profile. skin friction, Nusselt and Sherwood number.

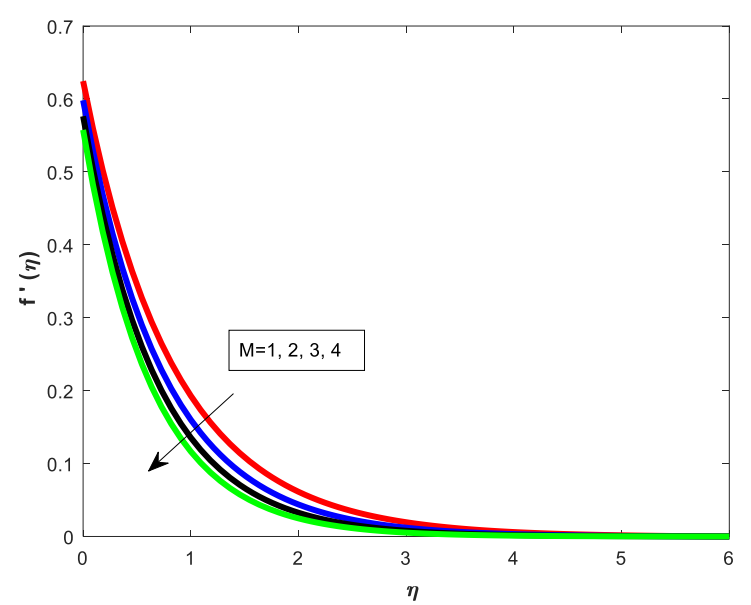

Figure 3: Effect of $\mathrm{M}$ on velocity profile.

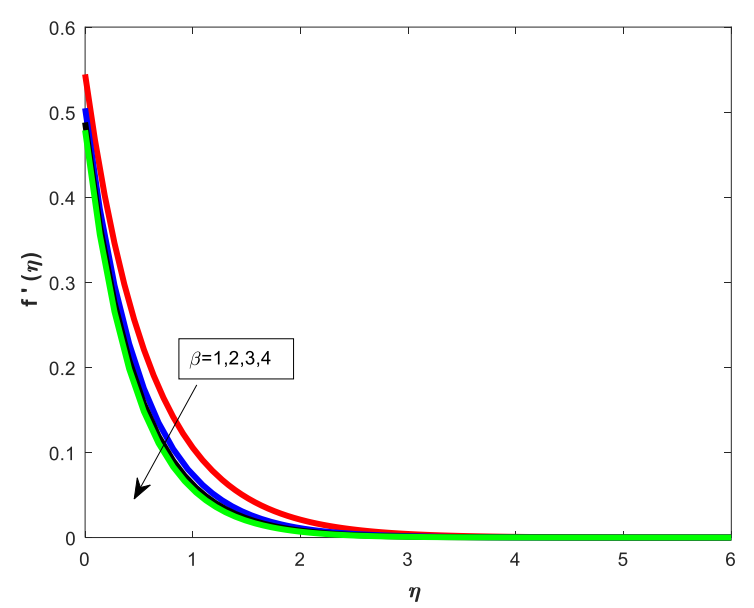

Figure 5: Effect of $\beta$ on velocity profile.

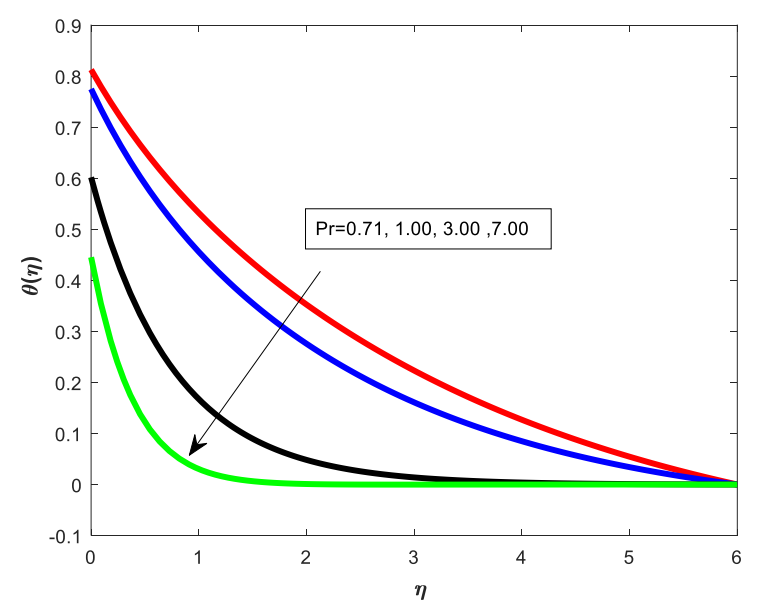

Figure 7: Effect of Pr on temperature profile 


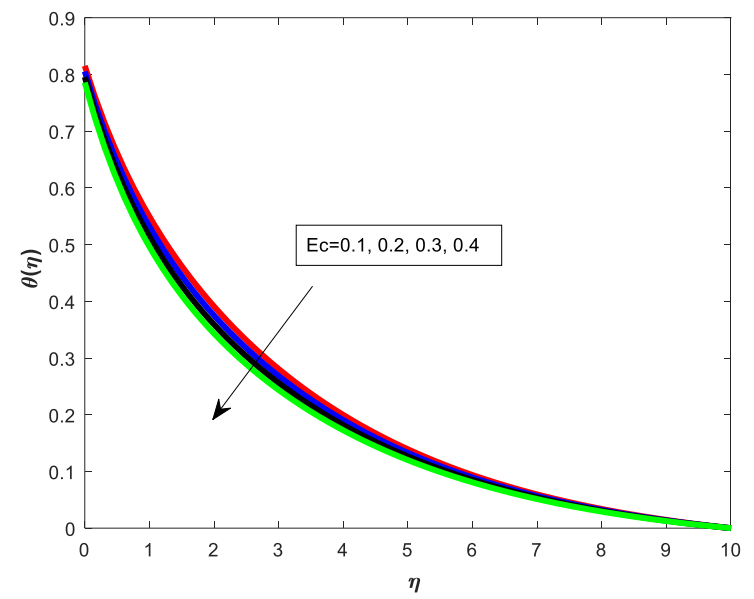

Figure 8: Effect of Ec on temperature profile

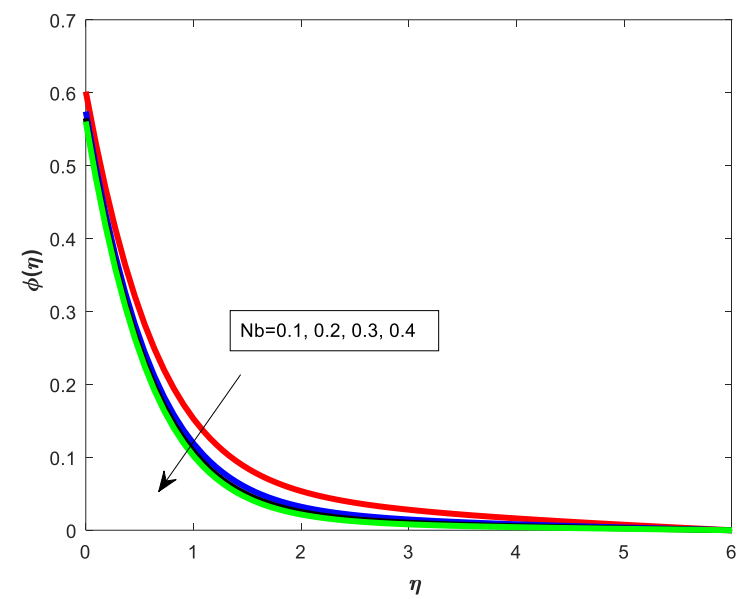

Figure 10: Effect of $\mathrm{Nb}$ on concentration profile.

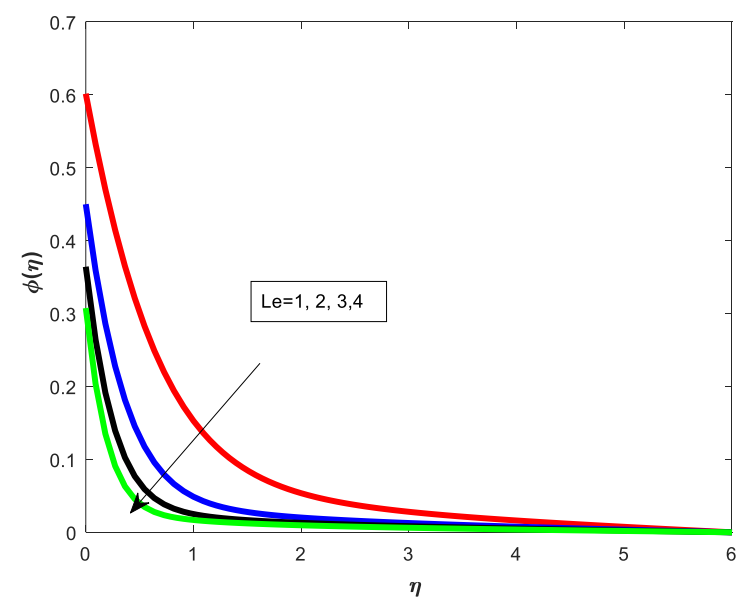

Figure 12: Effect of Le on concentration profile.

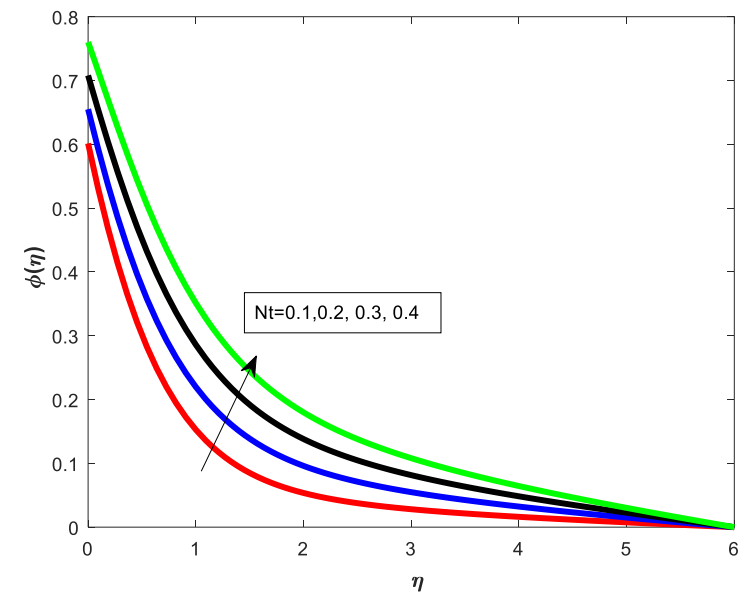

Figure 9: Effect of Nt on concentration profile

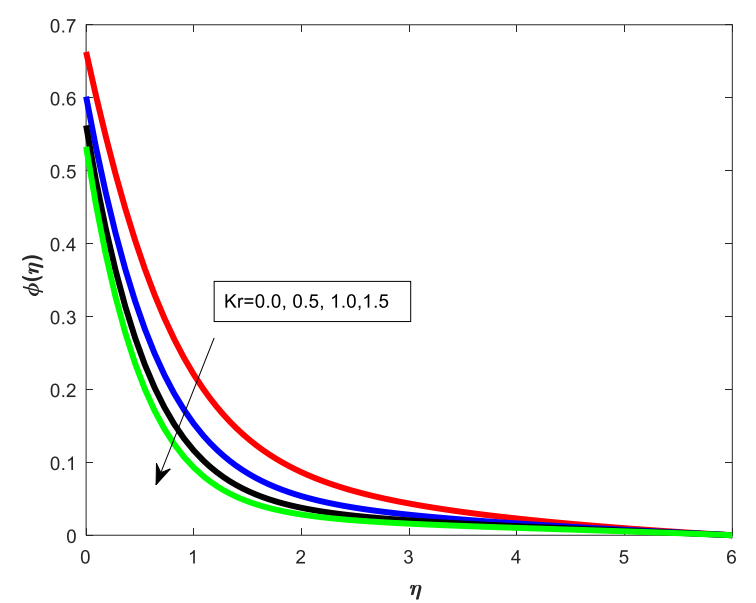

Figure 11: Effect of $\mathrm{Kr}$ on concentration profile.

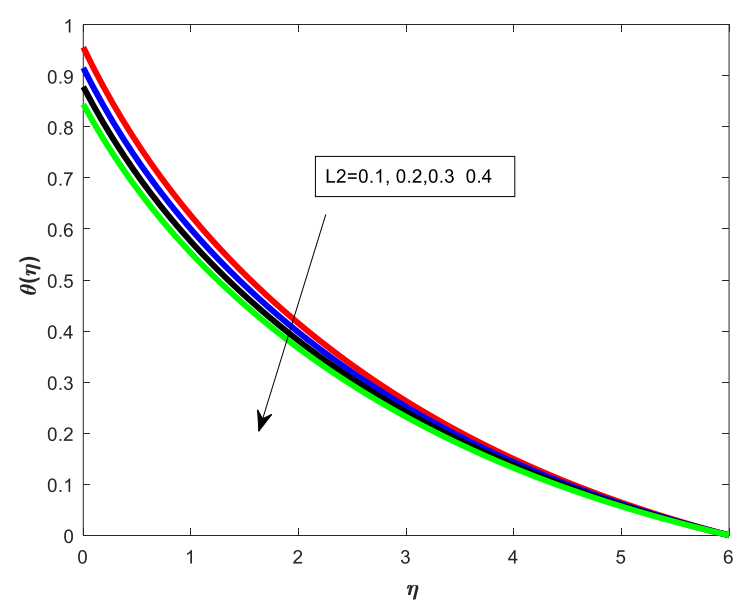

Figure 14: Effect of $L_{2}$ on temperature profile. 


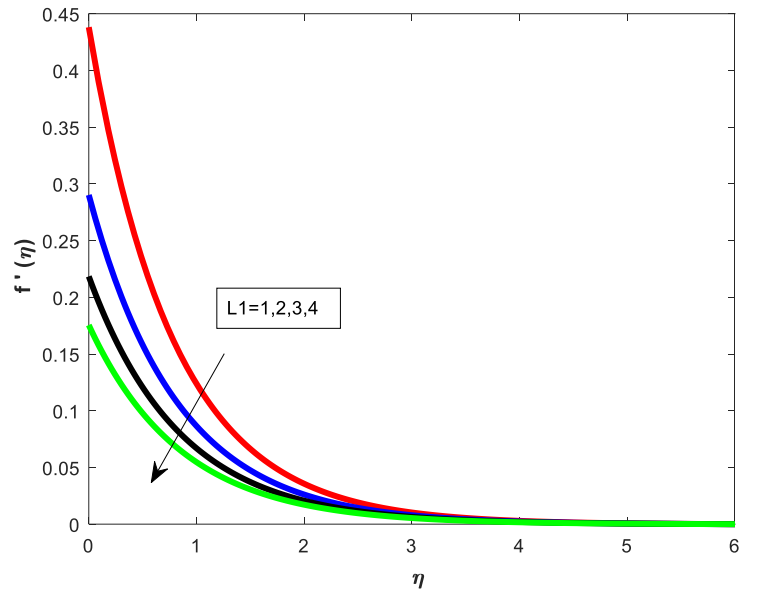

Figure 13: Effect of $\mathrm{L}_{1}$ on velocity profile.

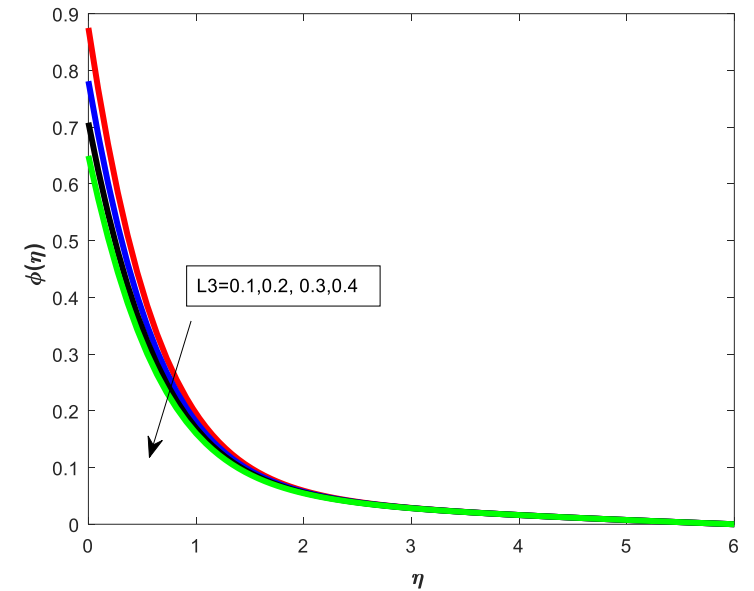

Figure 15: Effect of L3 on temperature profile.

Table 1: Numerical computational value of the skin friction, Nusselt and Sherwood numbers

\begin{tabular}{|c|c|c|c|c|c|c|c|c|c|c|c|c|c|c|c|c|c|}
\hline $\mathrm{M}$ & $\mathrm{Kr}$ & $\mathrm{n}$ & $\mathrm{B}$ & $\mathrm{R}$ & $\operatorname{Pr}$ & $\mathrm{Nt}$ & $\mathrm{Nb}$ & $\mathrm{Kr}$ & Ec & Le & $\mathrm{L}_{1}$ & $\mathrm{~L}_{2}$ & $\mathrm{~L}_{3}$ & $f_{w}$ & $\mathrm{Cf}$ & $\mathrm{Nu}$ & $\mathrm{Sh}$ \\
\hline 1 & 0.5 & 3 & 0.5 & 2 & 0.71 & 0.5 & 0.5 & 0.5 & 0.5 & 1 & 0.5 & 0.5 & 0.5 & 0.5 & 0.751056 & 0.576817 & 0.679085 \\
\hline 2 & & & & & & & & & & & & & & & 0.803672 & 0.591244 & 0.679420 \\
\hline 3 & & & & & & & & & & & & & & & 0.847451 & 0.607771 & 0.680427 \\
\hline 4 & & & & & & & & & & & & & & & 0.884901 & 0.626931 & 0.682576 \\
\hline \multirow[t]{4}{*}{2} & 1 & 3 & 0.5 & 2 & 0.71 & 0.5 & 0.5 & 0.5 & 0.5 & 1 & 0.5 & 0.5 & 0.5 & 0.5 & 0.826480 & 0.558263 & 0.679084 \\
\hline & 2 & & & & & & & & & & & & & & 0.866853 & 0.570261 & 0.679188 \\
\hline & 3 & & & & & & & & & & & & & & 0.901764 & 0.583796 & 0.679305 \\
\hline & 4 & & & & & & & & & & & & & & 0.932480 & 0.599216 & 0.679816 \\
\hline \multirow[t]{4}{*}{2} & 0.5 & 1 & 0.5 & 2 & 0.71 & 0.5 & 0.5 & 0.5 & 0.5 & 1 & 0.5 & 0.5 & 0.5 & 0.5 & 0.713950 & 0.456873 & 0.576210 \\
\hline & & 2 & & & & & & & & & & & & & 0.762119 & 0.541240 & 0.627048 \\
\hline & & 3 & & & & & & & & & & & & & 0.803672 & 0.607771 & 0.680427 \\
\hline & & 4 & & & & & & & & & & & & & 0.840360 & 0.662826 & 0.732884 \\
\hline \multirow[t]{4}{*}{2} & 0.5 & 3 & 1 & 2 & 0.71 & 0.5 & 0.5 & 0.5 & 0.5 & 1 & 0.5 & 0.5 & 0.5 & 0.5 & 0.911101 & 0.456803 & 0.698083 \\
\hline & & & 2 & & & & & & & & & & & & 0.990830 & 0.466261 & 0.709069 \\
\hline & & & 3 & & & & & & & & & & & & 1.024158 & 0.484212 & 0.713204 \\
\hline & & & 4 & & & & & & & & & & & & 1.042564 & 0.531783 & 0.715379 \\
\hline \multirow[t]{4}{*}{2} & 0.5 & 3 & 0.5 & 1 & 0.71 & 0.5 & 0.5 & 0.5 & 0.5 & 1 & 0.5 & 0.5 & 0.5 & 0.5 & 0.803672 & 0.467836 & 0.616139 \\
\hline & & & & 2 & & & & & & & & & & & 0.803672 & 0.525450 & 0.680427 \\
\hline & & & & 3 & & & & & & & & & & & 0.803672 & 0.607771 & 0.721610 \\
\hline & & & & 4 & & & & & & & & & & & 0.803672 & 0.734137 & 0.750228 \\
\hline \multirow[t]{4}{*}{2} & 0.5 & 3 & 0.5 & 5 & 0.71 & 0.1 & 0.1 & 0.5 & 0.001 & 1 & 0.5 & 0.5 & 0.5 & 0.5 & 0.803672 & 0.374465 & 0.404055 \\
\hline & & & & & 1 & & & & & & & & & & 0.803672 & 0.450143 & 0.582207 \\
\hline & & & & & 3 & & & & & & & & & & 0.803672 & 0.796291 & 0.759728 \\
\hline & & & & & 7 & & & & & & & & & & 0.803672 & 1.109419 & 0.797016 \\
\hline \multirow[t]{4}{*}{2} & 0.5 & 3 & 0.5 & 5 & 0.71 & 0.1 & 0.1 & 0.5 & 0.001 & 1 & 0.5 & 0.5 & 0.5 & 0.5 & 0.803672 & 0.370300 & 0.479406 \\
\hline & & & & & & 0.2 & & & & & & & & & 0.803672 & 0.371681 & 0.583944 \\
\hline & & & & & & 0.3 & & & & & & & & & 0.803672 & 0.373069 & 0.689810 \\
\hline & & & & & & 0.4 & & & & & & & & & 0.803672 & 0.374465 & 0.797016 \\
\hline \multirow[t]{3}{*}{2} & 0.5 & 3 & 0.5 & 5 & 0.71 & 0.1 & 0.1 & 0.5 & 0.001 & 1 & 0.5 & 0.5 & 0.5 & 0.5 & 0.803672 & 0.369399 & 0.797016 \\
\hline & & & & & & & 0.2 & & & & & & & & 0.803672 & 0.371081 & 0.851745 \\
\hline & & & & & & & 0.3 & & & & & & & & 0.803672 & 0.372770 & 0.869987 \\
\hline
\end{tabular}




\begin{tabular}{|c|c|c|c|c|c|c|c|c|c|c|c|c|c|c|c|c|c|}
\hline & & & & & & & 0.4 & & & & & & & & 0.803672 & 0.374465 & 0.879107 \\
\hline \multirow[t]{4}{*}{2} & 0.5 & 3 & 0.5 & 5 & 0.71 & 0.1 & 0.1 & 0 & 0.001 & 1 & 0.5 & 0.5 & 0.5 & 0.5 & 0.803672 & 0.374433 & 0.674408 \\
\hline & & & & & & & & 0.5 & & & & & & & 0.803672 & 0.374465 & 0.797016 \\
\hline & & & & & & & & 1 & & & & & & & 0.803672 & 0.374500 & 0.876119 \\
\hline & & & & & & & & 1.5 & & & & & & & 0.803672 & 0.374534 & 0.934493 \\
\hline \multirow[t]{4}{*}{2} & 0.5 & 3 & 0.5 & 5 & 0.71 & 0.1 & 0.1 & 0.5 & 0.1 & 1 & 0.5 & 0.5 & 0.5 & 0.5 & 0.803669 & 0.369696 & 0.760926 \\
\hline & & & & & & & & & 0.2 & & & & & & 0.803669 & 0.389295 & 0.770419 \\
\hline & & & & & & & & & 0.3 & & & & & & 0.803669 & 0.408891 & 0.779913 \\
\hline & & & & & & & & & 0.4 & & & & & & 0.803669 & 0.428484 & 0.789409 \\
\hline \multirow[t]{4}{*}{2} & 0.5 & 3 & 0.5 & 5 & 0.71 & 0.1 & 0.1 & 0.5 & 0.1 & 1 & 0.5 & 0.5 & 0.5 & 0.5 & 0.803672 & 0.374465 & 0.797016 \\
\hline & & & & & & & & & & 2 & & & & & 0.803672 & 0.374677 & 1.100114 \\
\hline & & & & & & & & & & 3 & & & & & 0.803672 & 0.374875 & 1.271602 \\
\hline & & & & & & & & & & 4 & & & & & 0.803672 & 0.375030 & 1.384959 \\
\hline \multirow[t]{4}{*}{2} & 0.5 & 3 & 0.5 & 5 & 0.71 & 0.1 & 0.1 & 0.5 & 0.1 & 1 & 1 & 0.5 & 0.5 & 0.5 & 0.206113 & 0.275373 & 0.780674 \\
\hline & & & & & & & & & & & 2 & & & & 0.260518 & 0.287149 & 0.781959 \\
\hline & & & & & & & & & & & 3 & & & & 0.354887 & 0.305830 & 0.784364 \\
\hline & & & & & & & & & & & & & & & 0.561871 & 0.340852 & 0.790082 \\
\hline \multirow[t]{4}{*}{2} & 0.5 & 3 & 0.5 & 5 & 0.71 & 0.1 & 0.1 & 0 & 0.1 & 1 & 1 & 1 & 0.5 & 0.5 & 0.206113 & 0.275373 & 0.780674 \\
\hline & & & & & & & & & & & & 2 & & & 0.260518 & 0.287149 & 0.781959 \\
\hline & & & & & & & & & & & & 3 & & & 0.354887 & 0.305830 & 0.784364 \\
\hline & & & & & & & & & & & & 4 & & & 0.561871 & 0.340852 & 0.790082 \\
\hline \multirow[t]{4}{*}{2} & 0.5 & 3 & 0.5 & 5 & 0.71 & 0.1 & 0.1 & 0 & 0.1 & 1 & 1 & 0.5 & 0.1 & 0.5 & 0.803672 & 0.388899 & 0.778229 \\
\hline & & & & & & & & & & & & & 0.2 & & 0.803672 & 0.404485 & 0.783510 \\
\hline & & & & & & & & & & & & & 0.3 & & 0.803672 & 0.421366 & 0.788370 \\
\hline & & & & & & & & & & & & & 0.4 & & 0.803672 & 0.439710 & 0.792859 \\
\hline \multirow[t]{4}{*}{2} & 0.5 & 3 & 0.5 & 5 & 0.71 & 0.1 & 0.1 & 0 & 0.1 & 1 & 1 & 0.5 & 0.5 & 0.1 & 0.803672 & 0.373617 & 0.876465 \\
\hline & & & & & & & & & & & & & & 0.2 & 0.803672 & 0.373908 & 0.973508 \\
\hline & & & & & & & & & & & & & & 0.3 & 0.803672 & 0.374134 & 1.094716 \\
\hline & & & & & & & & & & & & & & 0.4 & 0.803672 & 0.374316 & 1.250397 \\
\hline
\end{tabular}

\section{Conclusion}

The study at hand provides the numerical outcomes for a steady, viscous flow of Casson liquid alongside heat transport past an elongated sheet with thermal radiation and chemical reaction. The velocity decreases significantly owning to increase in Casson term. The temperature also degenerate significantly owning to increase in Prandtl number. The effect of increasing values of the magnetic term is to suppress the velocity, whereas the temperature is enhanced with increasing thermal radiation parameter. Prandtl number can be used to increase the rate of cooling in conducting flows.

\section{References}

[1] G Mahanta, S Shaw. 3D Casson fluid flow past a porous linearly stretching sheet with convective boundary condition, Alexandria Engineering Journal 2015; 54, 653-659.

[2] S P Samrat, CSulochana, G PAshwinkuma., Impact of Thermal Radiation on an Unsteady Casson Nanofluid Flow Over a Stretching Surface, International Journal of Applied and Computational Mathematics 2019, 5:31 https://doi.org/10.1007/s40819-019-0606$\underline{2}$

[3] Raza Jawad, Mushayydha Farooq, FatehMebarek-oudina, B. Mahanthesh. Multiple slip effects on MHD nonNewtonian nanofluid flow over a nonlinear permeable elongated sheet, Multidiscipline 
Modeling in Materials and Structures 2019; 15(5), 913-931.

[4] Swati Mukhopadhyay, PrativaRanjan De, Krishnendu Bhattacharyya, G.C. Layek.Casson fluid flow over an unsteady stretching surface,Ain Shams Engineering Journal 2013;4, 933-938.

[5] S Nadeem, RizwanUlHaq, C Lee. MHD flow of a Casson fluid over an exponentially shrinking sheet, ScientiaIranica B 2012; 19 (6), 1550-1553

[6] K Vijaya, G G R Reddy.Magnetohydrodynamic casson fluid flow over a vertical porous plate in the presence of radiation, soret and chemical reaction effects, Journal of Nanofluids, 2019; 8(6), 1240-1248.

[7] Y H Krishna, G V R Reddy, O D Makinde. Chemical reaction effect on MHD flow of casson fluid with porous stretching sheet, Defect and Diffusion Forum ,2018; 389, 100-109.

[8] A S Idowu, B O Falodun.Variable thermal conductivity and viscosity effects on nonNewtonian fluids flow through a vertical porous plate under Soret-Dufour influence, Mathematics and Computers in Simulation , 2020; 177, 358-384.

[9] S Idowu and B OFalodun. Effects of thermophoresis, Soret-Dufour on heat and mass transfer flow of magnetohydrodynamics non-Newtonian nanofluid over an inclined plate, Arab Journal of Basic and Applied Sciences, 2020, 27(1), 149-165..

[10] S Idowu Amos, T MojeedAkolade, Jos U. Abubakarand Bidemi $\mathrm{O}$ Falodun. MHD free convective heat and mass transfer flow of dissipative Casson fluid with variable viscosity and thermal conductivity effects, Journal of Taibah University for Science, 2020, 14(1) 851862 , DOI: 10.1080/16583655.2020.1781431.

[11] MangleshAarti and M. G. Gorla. The Effects of Thermal Radiation, Chemical Reaction and Rotation on Unsteady MHD Viscoelastic Slip Flow, Global Journal of Science Frontier Research Mathematics and Decision Sciences,2012; 12, 1-15.
[12] Ullah,K Bhattacharyya, S Shafie, I Khan. Unsteady MHD Mixed Convection Slip Flowof Casson Fluid over Nonlinearly Stretching Sheet Embedded in a Porous Medium with Chemical Reaction, Thermal Radiation, Heat Generation/ Absorption and Convective Boundary Conditions. PLoS ONE, 2016, 11(10). doi:10.1371/journal.pone.0165348

[13] PBalaAnki Reddy, Magnetohydrodynamic flow of a Casson fluid over an exponentially inclined permeable stretching surface with thermal radiation and chemical reaction, Ain Shams Engineering Journal 2016;7, 593602.

[14] S M Ibrahim, F Mabood, K Suneetha, G Lorenzini.Effects of chemical reaction on combined heat and mass transfer by laminar mixed convection flow from vertical surface with induced magnetic field and radiation, Journal of Engineering Thermophysics,2017;26(2), 234-255.

[15] N Vedavathi, G Dharmaiah, K S Balamurugan, J Prakash.Heat transfer on mhd nanofluid flow over a semi infinite flat plate embedded in a porous medium with radiation absorption, heat source and diffusion thermo effect, Frontiers in Heat and Mass Transfer,2017;9(38), 1-8

[16] T Hymavathi, W Sridhar.Numerical study of flow and heat transfer of casson fluid over an exponentially porous stretching surface in presence of thermal radiation, International Journal of Mechanical and Production Engineering Research and Development,2018; 8(4), 1145-1154

[17] H Talla, P Kumari, W Sridhar.Numerical study to diffusion of chemically reactive species over MHD exponentially stretching surface of a casson fluid, International Journal of Mechanical Engineering and Technology,2018; 9(10), 470-481.

[18] P R Kumari, D Sree Devi.Effect of radiation and radiation absorption on convective heat and mass transfer flow of 
a viscous electrically conducting fluid in a non-uniformly heated vertical channel, International Journal of Mechanical and Production Engineering Research and Development,2018; 8(7), 1382-1390.

[19] V Arundhati, K V Chandra Sekhar, D R V Prasada Rao, G Sreedevi.Non-darcy convective heat and mass transfer flow through a porous medium in vertical channel with soret, dufour and chemical reaction effects, JP Journal of Heat and Mass Transfer,2018; 15(2), 213-240.

[20] K V Chandra Sekhar. Heat transfer analysis of second grade fluid over a stretching sheet through porous medium under the influence of chemical reaction parameter, International Journal of Mechanical and Production Engineering Research and Development,2018; 8(1), 605-612.

[21] K V Chandra Sekhar. MHD free convective heat and mass transfer flow past an accelerated vertical plate through a porous medium with hall current, rotation and soret effects, International Journal of Mechanical and Production Engineering Research and Development,2018; 8(3), 685-706.

[22] J R Konda, N P Madhusudhana, R Konijeti.MHD mixed convection flow of radiating and chemically reactive Casson nanofluid over a nonlinear permeable stretching sheet with viscous dissipation and heat source, Multidiscipline Modeling in Materials and Structures2018; 14(3), 609-630.

[23] N Vijaya., M R Madhavi, Y H Krishna.Boundary layer flow of a mixed convective nanofluid over a vertical circular cylinder under the influence of magnetic field, heat radiation and external surface temperature, International Journal of Mechanical and Production Engineering Research and Development. 2018; 8,411-420.

[24] K Suneetha, S M Ibrahim, G V Ramana Reddy, A study on free convective heat and mass transfer flow through a highly porous medium with radiation, chemical reaction and soret effects, Journal of Computational and Applied Research in Mechanical Engineering,2019,8(2), 121132.

[25] F I Alao, A I Fagbade, B OFalodun, Effects of thermal radiation, Soret and Dufour on an unsteady heat and mass transfer flow of a chemically reacting fluid past a semi-infinite vertical plate with viscous dissipation, Journal of the Nigerian Mathematical Society.2016;35 (2016) 142-158.

[26] S Idowu, B O Falodun, Influence of Magnetic Field and Thermal Radiation on Steady Free Convective Flow in a Porous Medium, Nigerian journal of technological development, 2018; 15 (3), 84-97.

[27] A I Fagbade, B O Falodun, A J Omowaye. MHD natural convection flow of viscoelastic fluid over an accelerating permeable surface with thermal radiation and heat source or sink: Spectral Homotopy Analysis Approach, Ain Shams Engineering Journal 2018;9, 1029-1041. 